\title{
Strong Direct Product Theorems for Quantum Communication and Query Complexity*
}

\author{
Alexander A. Sherstov \\ Microsoft Research \\ 1 Memorial Drive \\ Cambridge, MA 02142 USA \\ sherstov@alumni.cs.utexas.edu
}

\begin{abstract}
A strong direct product theorem (SDPT) states that solving $n$ instances of a problem requires $\Omega(n)$ times the resources for a single instance, even to achieve success probability $2^{-\Omega(n)}$. We prove that quantum communication complexity obeys an SDPT whenever the communication lower bound for a single instance is proved by the generalized discrepancy method, the strongest technique in that model. We prove that quantum query complexity obeys an SDPT whenever the query lower bound for a single instance is proved by the polynomial method, one of the two main techniques in that model. In both models, we prove the corresponding XOR lemmas and threshold direct product theorems.
\end{abstract}

\section{Categories and Subject Descriptors}

F.0 [Theory of Computation]: General; F.1.3 [Computation by Abstract Devices]: Complexity Measures and Classes

\section{General Terms}

Theory

\section{Keywords}

Strong direct product theorems, XOR lemmas, quantum communication complexity, quantum query complexity

\section{INTRODUCTION}

A natural question to ask of any computational model is how the resources needed to solve $n$ instances of a problem scale with $n$. More concretely, suppose that solving a single instance of a given decision problem, with probability of correctness $4 / 5$, requires $R$ units of a computational resource (such as time, memory, communication, or queries). How many units of the resource are needed to solve $n$ independent instances of the problem? Common sense suggests that the answer should be $\Omega(n R)$. After all, having less than $\epsilon n R$ units overall, for a small constant $\epsilon>0$, leaves less than $\epsilon R$

\footnotetext{
${ }^{*}$ For a full version of this paper with complete proofs, see [41].
}

Permission to make digital or hard copies of all or part of this work for personal or classroom use is granted without fee provided that copies are not made or distributed for profit or commercial advantage and that copies bear this notice and the full citation on the first page. To copy otherwise, to republish, to post on servers or to redistribute to lists, requires prior specific permission and/or a fee.

STOC'11, June 6-8, 2011, San Jose, California, USA

Copyright 2011 ACM 978-1-4503-0691-1/11/06 ...\$10.00. units per instance, forcing the algorithm to guess random answers for many of the instances and resulting in overall success probability $2^{-\Omega(n)}$. Such a statement is called a strong direct product theorem. A related notion is an XOR lemma, which asserts that computing the XOR of the answers to the $n$ problem instances requires $\Omega(n R)$ resources, even if one is willing to settle for a success probability of $1 / 2+2^{-\Omega(n)}$. While highly plausible, XOR lemmas and strong direct product theorems are notoriously hard to prove and sometimes flat out wrong. To a considerable extent, the difficulty stems from the claimed exponential decay in the probability of successful computation. Dropping this part of the claim from strong direct product theorems results in direct sum theorems, which nevertheless are also elusive.

Apart from their inherent importance in theoretical computer science, direct product-type results have various applications, including separations of circuit classes [23], improvement of soundness in proof systems [36], inapproximability results for optimization problems [9], [18], and time-space trade-offs [27], [4]. Perhaps the two most famous results in this line of research are Yao's XOR lemma [48] for circuits, which was in 1982 the first result of the kind, and Raz's parallel repetition theorem [36] for twoprover games. Considerable progress has been achieved in these and various other models, complemented by surprising counterexamples [17], [38], [6]. The models of interest to us in this paper are quantum communication complexity and quantum query complexity, where the direct product phenomenon is understood quite poorly. Furthermore, work here has advanced much more slowly than in the classical case, a point conveyed by the following overview of the classical and quantum literature.

Classical communication and query complexity. The direct sum problem in communication complexity was raised for the first time in the work of Karchmer, Raz, and Wigderson [23], who showed that its resolution for relations would yield an explicit function outside $\mathrm{NC}^{1}$. Feder, Kushilevitz, Naor, and Nisan [17] established a direct sum theorem for nondeterministic communication complexity and inferred a weaker result for deterministic communication. Information-theoretic methods have enabled substantial progress [14], [5], [21], [22], [19], [6] on the direct sum question in the randomized model and its restrictions, including one-way communication and simultaneous message passing. In what generality randomized communication complexity obeys a direct sum theorem remains unknown; some counterexamples have been discovered for a careful choice of parameters [17].

It also remains unknown whether randomized communication complexity in general obeys a strong direct product theorem. A variety of results have been established, however, for concrete functions and some restrictions of the randomized model. Parnafes, Raz, and Wigderson [35] proved the first result of the kind, for 
"forests" of communication protocols. Shaltiel [38] proved an XOR lemma for uniform-distribution discrepancy, a well-studied communication complexity measure. Shaltiel's result has been generalized and strengthened in several ways [24], [8], [45], [31]. Jain, Klauck, and Nayak [20] obtained strong direct product theorems for an information-theoretic complexity measure called the $s u b$ distribution bound. Most recently, Klauck [26] proved the longconjectured strong direct product theorem for the randomized communication complexity of the disjointness function.

In classical query complexity, the direct product phenomenon is well understood. Strong direct product theorems have been obtained for "decision forests" by Nisan, Rudich, and Saks [34], for "fair" decision trees by Shaltiel [38], and for the randomized query complexity of symmetric functions by Klauck, Špalek, and de Wolf [27]. Recently, Drucker [16] obtained strong direct product theorems for the randomized query complexity of all functions.

Quantum communication and query complexity. Klauck, Špalek, and de Wolf [27] proved a strong direct product theorem for the quantum communication complexity of the disjointness function. Shaltiel [38] and Lee, Shraibman, and Špalek [31] obtained an XOR lemma for correlation with low-cost protocols, which gives a strong direct product theorem for certain communication problems such as Hadamard matrices or random matrices. The only other results known to us are for the alternate models of one-way communication and simultaneous message passing [22], [10], [19].

The results for quantum query complexity are just as few in number. The first direct product result is due to Aaronson [1], who proved it for the problem of $k$-fold search. Aaronson's result was improved to optimal with respect to all parameters by Klauck, Špalek, and de Wolf [27], who established a strong direct product theorem for the quantum query complexity of the OR function. In follow-up work, Ambainis, Špalek, and de Wolf [4] obtained a strong direct product theorem for all other symmetric functions. The only other result of which we are aware is due to Špalek [44], who developed a multiplicative version of the adversary method for quantum query complexity and proved that it obeys a strong direct product theorem.

\subsection{Our results}

In what follows, the symbol $f^{\otimes n}$ refers to the XOR of $n$ independent copies of a given decision problem $f$, which is a sign matrix in the case of communication complexity and a Boolean function $f:\{-1,+1\}^{m} \rightarrow\{-1,+1\}$ in the case of query complexity. The symbol $f^{(n)}$ refers to the task of simultaneously solving $n$ independent instances of $f$. In the latter context, we recall the notion of a threshold direct product theorem, which is a stronger statement than a strong direct product theorem. Specifically, a threshold direct product theorem defines successful computation of $f^{(n)}$ as correct computation of $(1-\beta) n$ instances for a small constant $\beta>0$, as opposed to correct computation of all $n$ instances. A threshold direct product theorem states that computing $n$ instances requires $\Omega(n)$ times the resources for a single instance, even to achieve success probability $2^{-\Omega(n)}$ with this relaxed criterion of correct computation. All our direct product theorems are threshold direct product theorems.

Quantum communication. Let $\mathscr{R}$ denote the family of $0 / 1$ matrices in which the 1 entries form a submatrix. Such matrices are called rectangles and are the basic building blocks in communication complexity. In particular, the matrix $\Pi$ of acceptance probabilities of any communication protocol with cost $c$ obeys

$$
\Pi \in 2^{O(c)} \operatorname{conv}\{ \pm R: R \in \mathscr{R}\} .
$$

This fact has an elementary and well-known demonstration [29] for classical protocols. The validity of (1.1) for quantum protocols, on the other hand, was open for several years and settled relatively recently in an elegant paper of Linial and Shraibman [33]. This fact immediately gives a criterion for high communication complexity, known as the generalized discrepancy method. Specifically, define a norm $\mu$ on matrices by letting $\mu(\Pi)$ be the least $K \geqslant 0$ for which $\Pi \in K \operatorname{conv}\{ \pm R: R \in \mathscr{R}\}$. Then a sign matrix has high boundederror communication complexity whenever every real matrix in its neighborhood has high $\mu$ norm. The method has an equivalent $d u a l$ formulation that is widely used and has a rich history, e.g., [25], [37], [33], [40], [42], [30].

Linial and Shraibman [33] showed that the generalized discrepancy method subsumes all earlier criteria for high quantum communication complexity. In particular, all known lower bounds for two-way quantum communication can be derived using the generalized discrepancy method and no additional facts about quantum mechanics. Furthermore, the full power of the method is rarely necessary, and the main lower bounds have all been obtained using a simpler criterion known as the trace norm method, e.g., [49], [28], [25], [37], [40], [43].

Our main result is that the generalized discrepancy method obeys an XOR lemma and a threshold direct product theorem. This solves an open problem posed in [31, Sec. 6]. In particular, whenever the generalized discrepancy method yields a tight lower bound on the quantum communication complexity of a sign matrix $F$ (as it does for all known $F$ ), one immediately obtains an XOR lemma and threshold direct product theorem for $F$. In what follows, we let the real number $\operatorname{GDM}_{\epsilon}(F)$ denote the lower bound that the generalized discrepancy method gives on the $\epsilon$-error quantum communication complexity of $F$.

THEOREM 1.1. Fix a sign matrix $F$. Then the following tasks require $\Omega\left(n \mathrm{GDM}_{1 / 5}(F)\right)$ qubits of communication each:

- solving $F^{\otimes n}$ with worst-case probability $1 / 2+2^{-\Omega(n)}$;

- solving $F^{(n)}$ with worst-case probability $2^{-\Omega(n)}$.

The same holds for solving with probability $\geqslant 2^{-\dot{\Omega}(n)}$ at least $(1-\beta) n$ among $n$ instances of $F$, for small $\beta>0$.

It is natural to consider the direct product question in the broader context of distinct communication problems $F_{1}, F_{2}, \ldots, F_{n}$, rather than $n$ instances of the same communication problem. This paper gives a detailed solution in the generalized setting as well. As before, we consider the task of computing the XOR of the answers, denoted $F_{1} \otimes \cdots \otimes F_{n}$, and the task of solving each of the $n$ problems, denoted $\left(F_{1}, \ldots, F_{n}\right)$. Here, one clearly cannot hope to prove that $\Omega\left(\sum_{i=1}^{n} \operatorname{GDM}_{1 / 5}\left(F_{i}\right)\right)$ is a communication lower bound for solving the above two tasks with advantage $2^{-\Omega(n)}$ over random guessing. Indeed, if $F_{1}$ has communication cost larger than the other problems combined, then for all intents and purposes we are working with a single problem, and no exponential decay in success probability is possible by definition. But it is reasonable to expect a direct sum theorem here-and we prove that it indeed holds:

THEOREM 1.2. For all sign matrices $F_{1}, F_{2}, \ldots, F_{n}$, computing $F_{1} \otimes \cdots \otimes F_{n}$ with probability $4 / 5$ requires a communication protocol with cost $\Omega\left(\sum_{i=1}^{n} \operatorname{GDM}_{1 / 5}\left(F_{i}\right)\right)$.

We complement Theorem 1.2 by proving that a quantum protocol's success probability does indeed become exponentially close to that of random guessing when the protocol's communication is bounded by the sum of the smallest $\lceil 0.99 n\rceil$ of the numbers $\operatorname{GDM}_{1 / 5}\left(F_{1}\right), \ldots, \operatorname{GDM}_{1 / 5}\left(F_{n}\right)$ : 
THEOREM 1.3. Fix sign matrices $F_{1}, F_{2}, \ldots, F_{n}$ of rank greater than 1 . Then the following tasks have quantum communication cost $\Omega\left(\min _{|S|=\lceil 0.99 n\rceil}\left\{\sum_{i \in S} \operatorname{GDM}_{1 / 5}\left(F_{i}\right)\right\}\right)$ :

- solving $\bigotimes_{i=1}^{n} F_{i}$ with worst-case probability $1 / 2+2^{-\Omega(n)}$;

- solving $\left(F_{1}, \ldots, F_{n}\right)$ with worst-case probability $2^{-\Omega(n)}$.

The same holds for solving with probability $\geqslant 2^{-\Omega(n)}$ at least $(1-\beta) n$ among the $n$ instances, for small $\beta>0$.

All the theorems above are valid for quantum protocols with arbitrary prior entanglement. While stated above for worst-case complexity, Theorems 1.1-1.3 hold for average-case complexity under a certain joint probability distribution defined explicitly in our proof. Finally, we prove results identical to Theorems $1.1-1.3$ in the setting of partial communication problems, whose domain of definition is a proper subset of all possible inputs. In such cases $\operatorname{GDM}_{\epsilon}(F)$ is computed by considering the smallest $\mu$ norm over real matrices whose entries are within $\epsilon$ of the values of $F$ on the domain of $F$ and anywhere in $[-1-\epsilon, 1+\epsilon]$ outside the domain.

Quantum query complexity. The polynomial method, discovered by Beals, Buhrman, Cleve, Mosca, and de Wolf [7], is a technique for proving lower bounds on quantum query complexity. It is easy to state: The acceptance probability of a quantum query algorithm on input $x \in\{-1,+1\}^{m}$ is a real polynomial in $x_{1}, x_{2}, \ldots, x_{m}$ of degree at most $2 T$, where $T$ is the number of queries. Conversely, if there is no degree- $d$ real polynomial that approximates a given Boolean function $f$ within $1 / 5$ on all inputs, then $f$ has boundederror query complexity $\Omega(d)$. Beals et al. [7] used this method to obtain tight lower bounds on the query complexity of all symmetric functions. The polynomial method has since yielded many other tight lower bounds, e.g., [11], [2], [1], [27]. The main alternative to the polynomial method is the adversary method, introduced by Ambainis [3] and augmented in many subsequent works.

Our second main result is that the polynomial method obeys an XOR lemma and a threshold direct product theorem. In particular, whenever the polynomial method yields a tight lower bound on the query complexity of a given Boolean function $f$, one automatically obtains an XOR lemma and a threshold direct product theorem for $f$. This subsumes the functions $f$ in all previous direct product theorems for quantum query complexity [1], [27], [4]. As for communication complexity, we prove our results in the general setting of distinct functions $f_{1}, f_{2}, \ldots, f_{n}$ rather than $n$ instances of the same function $f$. In the statements to follow, the symbol $\operatorname{deg}_{\epsilon}(f)$ stands for the least degree of a real polynomial that approximates $f$ within $\epsilon$ pointwise.

THEOREM 1.4. For all functions $f_{1}, f_{2}, \ldots, f_{n}:\{-1,+1\}^{m} \rightarrow$ $\{-1,+1\}$, computing $f_{1} \otimes \cdots \otimes f_{n}$ with probability $4 / 5$ requires a quantum query algorithm with cost $\Omega\left(\sum_{i=1}^{n} \operatorname{deg}_{1 / 5}\left(f_{i}\right)\right)$.

We complement this with a direct product result analogous to the one for communication:

THEOREM 1.5. Fix $f_{1}, f_{2}, \ldots, f_{n}:\{-1,+1\}^{m} \rightarrow\{-1,+1\}$. The following tasks require $\Omega\left(\min _{|S|=\lceil 0.99 n\rceil}\left\{\sum_{i \in S} \operatorname{deg}_{1 / 5}\left(f_{i}\right)\right\}\right)$ quantum queries each:

- solving $\bigotimes_{i=1}^{n} f_{i}$ with worst-case probability $1 / 2+2^{-\Omega(n)}$;

- solving $\left(f_{1}, \ldots, f_{n}\right)$ with worst-case probability $2^{-\Omega(n)}$.

The same holds for solving with probability $\geqslant 2^{-\Omega(n)}$ at least $(1-\beta) n$ among the $n$ instances, for small $\beta>0$.

In particular, Theorem 1.5 shows that for every Boolean function $f$, the tasks of computing $f^{\otimes n}$ and $f^{(n)}$ each have quan- tum query complexity $\Omega\left(n \operatorname{deg}_{1 / 5}(f)\right)$, even to achieve advantage $2^{-\Omega(n)}$ over random guessing. The additional remarks made earlier in the context of communication carry over in full. Specifically, Theorems 1.4 and 1.5 remain valid for partial Boolean functions,

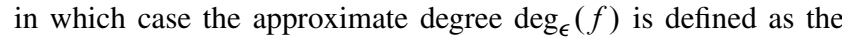
least degree of a polynomial that approximates $f$ within $\epsilon$ on the domain of $f$ and ranges freely in $[-1-\epsilon, 1+\epsilon]$ everywhere else on the hypercube. Lastly, Theorems 1.4 and 1.5 are stated worst-case complexity but are also valid for average-case complexity under a certain joint probability distribution given explicitly in our proof.

Consequences for polynomial approximation. In proving Theorem 1.5 , we show in particular that

$$
\operatorname{deg}_{1-2-\Omega(n)}\left(f^{\otimes n}\right) \geqslant \Omega\left(n \operatorname{deg}_{1 / 5}(f)\right) .
$$

To our knowledge, this is the first direct product theorem for polynomial approximation. It matches the upper bound due to Buhrman, Newman, Röhrig, and de Wolf [12, Thm. 6], who proved that $\operatorname{deg}_{1 / 5}\left(f^{\otimes n}\right)=O\left(n \operatorname{deg}_{1 / 5}(f)\right)$. We also obtain the first $d i$ rect sum results for polynomial approximation: given any function $F:\{-1,+1\}^{n} \rightarrow\{-1,+1\}$ with $\operatorname{deg}_{1 / 5}(F)=\Omega(n)$ (which includes the familiar majority and parity functions and the random functions), we prove that

$$
\operatorname{deg}_{1 / 5}(F(f, f, \ldots, f))=\Omega\left(\operatorname{deg}_{1 / 5}(F) \operatorname{deg}_{1 / 5}(f)\right),
$$

for all Boolean functions $f$. This lower bound matches the upper bound in [12].

\subsection{Our techniques}

The proof technique of this paper is quite general and applies to any bounded-error model of computation that admits a representation as a convex subset of a real linear space. Examples of convex subsets that naturally arise from a computational model include the unit ball of a norm and the linear span of a given set of functions. Both of these cases are treated in this paper: the former corresponds to communication complexity and the latter, to query complexity. For simplicity, we will focus on the former setting in this overview.

Here, one fixes a finite set $X$ and lets the space of real functions on $X$ and $X^{n}$ be normed by $\|\cdot\| \cdot \|$. The norm captures the complexity of exact computation, as measured in the relevant resource. In other words, functions that represent low-cost communication protocols and low-cost query algorithms will have small norm. The complexity of $\epsilon$-error computation for a given function $f: X \rightarrow\{-1,+1\}$ is then given by the minimum norm in the $\epsilon$-neighborhood of $f$. This norm-based formalism is particularly natural in quantum computing and has been in use for many years, e.g., [49], [28], [37].

XOR lemmas for correlation represent a particularly wellstudied form of hardness amplification in this setting: given a function $f: X \rightarrow\{-1,+1\}$ that has small correlation $\gamma$ with all lowcost communication protocols or low-cost query algorithms, one argues that for $f^{\otimes n}$ the correlation further drops to $\gamma^{\Omega(n)}$. In the language of norms, a function $f$ has small correlation with the simple functions if and only if the dual norm $\|f\|^{*}$ is small. Thus, an XOR lemma for correlation is an assertion about the multiplicativity of the dual norm: $\left\|f^{\otimes n}\right\|^{*} \leqslant\left(\|f\|^{*}\right)^{\Omega(n)}$. Much of the research surveyed above [38], [15], [45], [31] fits in this framework.

This paper addresses a rather different problem. While we also seek to establish XOR lemmas, we start with a much more general object: a function $f: X \rightarrow\{-1,+1\}$ with high bounded-error computational complexity. The key point is that $f$ need no longer have small dual norm $\|f\|^{*}$, or equivalently small correlation with the low-cost functions. Indeed, many common functions with nearmaximum bounded-error complexity, such as the OR function in 
query complexity and the disjointness function in communication complexity, have high correlation with the low-cost protocols and query algorithms under every distribution on the domain. Thus, the above research on XOR lemmas for correlation no longer applies.

This described difficulty crystallizes best in the language of norms. By duality, a given function $f: X \rightarrow\{-1,+1\}$ of interest has high bounded-error complexity if and only if there exists a real-valued function $\psi: X \rightarrow\{-1,+1\}$ of unit $\ell_{1}$ norm that has reasonably large inner product $\langle f, \psi\rangle$ but low dual norm $\|\psi\|^{*}$. This function $\psi$ is a witness to the fact that $f$ has high bounded-error complexity. The challenge is to construct a corresponding witness for $f^{\otimes n}$. The natural candidate, $\psi^{\otimes n}$, is completely useless for this purpose: while $\left\|\psi^{\otimes n}\right\|_{1}=1$ and moreover we can certainly hope for an exponential decay in the dual norm $\left\|\psi^{\otimes n}\right\|^{*} \leqslant\left(\|\psi\|^{*}\right)^{\Omega(n)}$, the correlation with $f^{\otimes n}$ will also decay exponentially: $\left\langle f^{\otimes}, \psi^{\otimes n}\right\rangle=\langle f, \psi\rangle^{n}$. This translates to an uninteresting statement like "computing $f^{\otimes n}$ with error probability $2^{-\Omega(n)}$ incurs $\Omega(n)$ times the cost of computing $f$." We want the opposite: the error probability allowed in computing $f^{\otimes n}$ should be exponentially close to the trivial rate $1 / 2$ rather than to 0 .

The crux of our solution is the construction of the sought witness for $f^{\otimes n}$, using ideas from approximation theory to design a joint, nonproduct distribution under which the correlation of $\psi^{\otimes}$ and $f^{\otimes}$ becomes extremely high but the dual norm $\left\|\psi^{\otimes}\right\|$ remains extremely low. This construction works for any norm whose dual $\|\cdot \cdot\|^{*}$ possesses a multiplicative property, as our norm of interest for which multiplicativity was established in previous work by Cleve, Slofstra, Unger, and Upadhyay [15].

This sketches some ideas in the proofs of the XOR lemmas. The direct product theorems are then derived by combining the XOR lemmas with known results on the low-error approximation of symmetric Boolean functions. In particular, we appeal to a result of de Wolf [47] that OR and other symmetric Boolean functions admit uniform approximation to within $2^{-\Omega(n)}$ by a polynomial of degree $\epsilon n$, for a small constant $\epsilon>0$.

\section{PRELIMINARIES}

We view Boolean functions as mappings $f: X \rightarrow\{-1,+1\}$ for some finite set $X$, where -1 and +1 correspond to "true" and "false," respectively. A partial Boolean function $g$ on a finite set $X$ is a mapping $g: D \rightarrow\{-1,+1\}$ for some nonempty proper subset $D \subset X$. We denote the domain of $g$ by $\operatorname{dom} g=D$. For emphasis, we will occasionally refer to Boolean functions with dom $g=X$ as total. For a string $x \in\{-1,+1\}^{n}$, we use the shorthand $|x|=\left|\left\{i: x_{i}=-1\right\}\right|=\sum\left(1-x_{i}\right) / 2$. For $\epsilon_{1}, \epsilon_{2}, \ldots, \epsilon_{n} \in[0,1]$, the symbol $\Pi\left(\epsilon_{1}, \epsilon_{2}, \ldots, \epsilon_{n}\right)$ stands for the probability distribution on $\{-1,+1\}^{n}$ whereby the $i$ th bit of the string takes on -1 with probability $\epsilon_{i}$, independently for each $i$. For an event $E$, the corresponding indicator function is $\mathbf{I}[E] \in\{0,1\}$. We adopt the following two versions of the sign function:

$$
\operatorname{sgn} t=\left\{\begin{array}{ll}
-1, & t<0, \\
0, & t=0, \\
1, & t>0 ;
\end{array} \quad \widetilde{\operatorname{sgn}} t= \begin{cases}-1, & t<0, \\
1, & t \geqslant 0 .\end{cases}\right.
$$

We will specify an $n$-bit string by its $i$ th bit, for example, $(\ldots,(\epsilon-$ $\left.\left.\epsilon_{i}\right) /\left(1-\epsilon_{i}\right), \ldots\right)$ or $\left(\ldots, z_{i}, \ldots\right)$. The Cartesian product of sets $X_{1}, X_{2}, \ldots, X_{n}$ is denoted $\prod X_{i}$, or for greater explicitness $X_{1} \times$ $X_{2} \times \cdots \times X_{n}$. The degree of a real polynomial $p$ is denoted deg $p$.

Given a function $\phi:\{-1,+1\}^{n} \rightarrow \mathbb{R}$, there exists a unique $m u l$ tilinear polynomial $\tilde{\phi}: \mathbb{R}^{n} \rightarrow \mathbb{R}$ such that $\phi \equiv \tilde{\phi}$ on $\{-1,+1\}^{n}$. We will always identify $\phi$ with its multilinear extension $\tilde{\phi}$ to $\mathbb{R}^{n}$. In particular, we will write $\phi(z)$ for arbitrary $z \in[-1,1]^{n}$.
For $n \geqslant k \geqslant 0$, we adopt the shorthand $\left(\begin{array}{c}n \\ \leqslant k\end{array}\right)=\sum_{i=0}^{k}\left(\begin{array}{c}n \\ i\end{array}\right)$. Throughout this manuscript, $\log x$ stands for the logarithm of $x$ to the base 2 .

Norms and duality. For a finite set $X$, the linear space of real functions on $X$ is denoted $\mathbb{R}^{X}$. This space is equipped with the usual norms and inner product:

$$
\left.\begin{array}{rlrl}
\|\phi\|_{\infty} & =\max _{x \in X}|\phi(x)| & & \left(\phi \in \mathbb{R}^{X}\right), \\
\|\phi\|_{1} & =\sum_{x \in X}|\phi(x)| & & \left(\phi \in \mathbb{R}^{X}\right), \\
\langle\phi, \psi\rangle & =\sum_{x \in X} \phi(x) \psi(x) & & \left(\phi, \psi \in \mathbb{R}^{X}\right) .
\end{array}\right\}
$$

The tensor product of $\phi \in \mathbb{R}^{X}$ and $\psi \in \mathbb{R}^{Y}$ is the function $\phi \otimes \psi \in$ $\mathbb{R}^{X \times Y}$ given by $(\phi \otimes \psi)(x, y)=\phi(x) \psi(y)$. The tensor product $\phi \otimes \phi \otimes \cdots \otimes \phi(n$ times $)$ is denoted $\phi^{\otimes n} \in \mathbb{R}^{X^{n}}$. When specialized to real matrices, the tensor product is the usual Kronecker product. The pointwise (Hadamard) product of functions $\phi, \psi \in \mathbb{R}^{X}$ is denoted $\phi \circ \psi \in \mathbb{R}^{X}$ and given by $(\phi \circ \psi)(x)=\phi(x) \psi(x)$. Note the difference between $\phi \otimes \psi$ and $\phi \circ \psi$.

For an arbitrary norm $\|\cdot\| \|$ on $\mathbb{R}^{X}$, recall that $\|\cdot\|^{*}$ refers to the dual norm given by $\|\phi\|^{*}=\max _{\psi \neq 0}\langle\phi, \psi\rangle /\|\psi\|$. A corollary to the duality $\|\cdot\|^{* *}=\|\cdot\| \|$ is the following classical fact pertaining to approximation; see [30, Thm. 6.3] and the full version of this paper [41].

FACT 2.1. Let $X$ be a finite set, let $N_{1}, N_{2}$ be norms on $\mathbb{R}^{X}$. Then for any $\epsilon \geqslant 0$ and any $f \in \mathbb{R}^{X}$ with $N_{2}(f)>\epsilon$,

$$
\min \left\{N_{1}(f-\xi): N_{2}(\xi) \leqslant \epsilon\right\}=\max _{\psi \neq 0} \frac{\langle f, \psi\rangle-\epsilon N_{2}^{*}(\psi)}{N_{1}^{*}(\psi)} .
$$

We will mainly be concerned with approximation in the infinity norm. This case is served by the notation $\|f\|_{\epsilon}=\min \{\|f-\xi\|$ : $\left.\|\xi\|_{\infty} \leqslant \epsilon\right\}$, where $f: X \rightarrow \mathbb{R}$ is a given function and $\|\cdot\| \cdot \|$ is a given norm on $\mathbb{R}^{X}$. When $f$ is a partial Boolean function on $X$, we define $\|f\| \epsilon$ to be the least norm $\|\phi\|$ over all elements $\phi \in \mathbb{R}^{X}$ such that $|f(x)-\phi(x)| \leqslant \epsilon$ for all $x \in \operatorname{dom} f$ and $|\phi(x)| \leqslant 1+\epsilon$ for all $x \notin \operatorname{dom} f$. When $\operatorname{dom} f=X$, this agrees with the earlier definition of the symbol $\|f\|_{\epsilon}$. Fact 2.1 has the following basic consequence (see the full version [41] for a proof):

Corollary 2.2. Let $X$ be a finite set, $\|\cdot\|$ a norm on $\mathbb{R}^{X}$. Then for every $\epsilon \in(0,1)$ and every (possibly partial) Boolean function $f$ on $X$, the quantity $\|f\|_{\epsilon}$ equals

$$
\max _{\psi \neq 0} \frac{1}{\|\psi\|^{*}}\left\{\sum_{x \in \operatorname{dom} f} f(x) \psi(x)-\sum_{x \notin \operatorname{dom} f}|\psi(x)|-\epsilon\|\psi\|_{1}\right\} .
$$

Matrix analysis. A special case covered by the notation (2.1) is the family $\mathbb{R}^{n \times m}$ of all real matrices of dimension $n \times m$. More explicitly, one has $\|A\|_{\infty}=\max \left|A_{i j}\right|,\|A\|_{1}=\sum\left|A_{i j}\right|$, and $\langle A, B\rangle=\sum A_{i j} B_{i j}$ for all $A, B \in \mathbb{R}^{n \times m}$. For finite sets $X$ and $Y$, we let $\mathbb{R}^{X \times Y}$ and $\{-1,+1\}^{X \times Y}$ stand for the families of real and \pm 1 matrices, respectively, with rows indexed by elements of $X$ and columns indexed by elements of $Y$. The rank of a matrix $A$ over the reals is denoted $\operatorname{rk} A$. The $\operatorname{symbol} \operatorname{diag}\left(a_{1}, a_{2}, \ldots, a_{n}\right)$ refers to the diagonal matrix of order $n$ with entries $a_{1}, a_{2}, \ldots, a_{n}$ on the diagonal. A signature scaling of a matrix $M \in \mathbb{R}^{n \times m}$ is any matrix of the form $\operatorname{diag}\left(a_{1}, \ldots, a_{n}\right) M \operatorname{diag}\left(b_{1}, \ldots, b_{m}\right)$, where $a_{1}, \ldots, a_{n}, b_{1}, \ldots, b_{m} \in\{-1,+1\}$. The symbols $I_{n}$ and 
$J_{n, m}$ refer to the identity matrix of order $n$ and the all-ones matrix of dimension $n \times m$, respectively; we will drop the subscripts and write $I, J$ whenever the dimension is clear from the context. A sign matrix is any matrix with entries \pm 1 . A Hadamard matrix is any sign matrix $A$ of order $n$ that obeys $A A^{\top}=n I$.

The Frobenius norm is given by $\|M\|_{\mathrm{F}}=\left(\sum M_{i j}^{2}\right)^{1 / 2}$. The singular values of $M$ are denoted $\sigma_{1}(M) \geqslant \sigma_{2}(M) \geqslant \sigma_{3}(M) \geqslant \cdots$, with the spectral norm and trace norm given by $\|M\|=\sigma_{1}(M)$ and $\|M\|_{\Sigma}=\sum \sigma_{i}(M)$, respectively. The spectral norm and trace norm are duals of each other. An equivalent definition of the trace norm is $\|M\|_{\Sigma}=\min \left\{\|A\|_{\mathrm{F}}\|B\|_{\mathrm{F}}: A B=M\right\}$. A close relative of the trace norm is the $\gamma_{2}$ norm, defined by $\gamma_{2}(M)=\min \left\{\|A\|_{\text {row }}\|B\|_{\text {col }}: A B=M\right\}$, where $\|A\|_{\text {row }}$ and $\|B\|_{\mathrm{col}}$ stand for the largest Euclidean norm of a row of $A$ and the largest Euclidean norm of a column of $B$, respectively. Put $\gamma_{2, \epsilon}(M)=\min \left\{\gamma_{2}(M-E):\|E\|_{\infty} \leqslant \epsilon\right\}$, the least $\gamma_{2}$ norm of a matrix in the $\epsilon$-neighborhood of $M$. Below, we collect some well-known properties of $\gamma_{2}$ (see the full version [41] for a proof).

FACT 2.3. Let $A$ and $B$ denote arbitrary real matrices. Let $H_{N}$ be a Hadamard matrix of order N. Then:

(i) $\gamma_{2}(A)=\gamma_{2}(B)$ whenever $A$ is a signature scaling of $B$,

(ii) $\gamma_{2}(A) \geqslant \gamma_{2}(B)$ whenever $B$ is a submatrix of $A$,

(iii) $\gamma_{2}$ is invariant under duplication of rows and columns,

(iv) $\gamma_{2}(A) \geqslant\|A\|_{\infty}$,

(v) $\gamma_{2}(A) \geqslant\|A\|_{\Sigma} / \sqrt{n m}$ for all $A \in \mathbb{R}^{n \times m}$,

(vi) $\gamma_{2}^{*}(A) \leqslant\|A\| \sqrt{n m}$ for all $A \in \mathbb{R}^{n \times m}$,

(vii) $\gamma_{2}(J)=1$,

(viii) $\gamma_{2}\left(H_{N}\right)=\sqrt{N}$,

(ix) $\gamma_{2, \epsilon}(A) \geqslant(1-\epsilon) \sqrt{n m} /\|A\|$ for all $A \in\{-1,+1\}^{n \times m}$,

(x) $\gamma_{2, \epsilon}(J)=1-\epsilon$ for $0 \leqslant \epsilon \leqslant 1$,

(xi) $\gamma_{2, \epsilon}\left(H_{N}\right)=(1-\epsilon) \sqrt{N}$ for $0 \leqslant \epsilon \leqslant 1$,

(xii) $\gamma_{2}(A \otimes B) \leqslant \gamma_{2}(A) \gamma_{2}(B)$,

(xiii) $\gamma_{2}(A \circ B) \leqslant \gamma_{2}(A) \gamma_{2}(B)$.

Tracing the authorship of the items in Fact 2.3 is challenging. Items (v) and (vi) appear in [32], and the others are likely classical.

In the context of lower bounds on communication complexity, we will encounter partial sign matrices, which are matrices with entries in $\{-1,+1, *\}$. For a partial sign matrix $F$ and a norm $\|\cdot\|$, we let $\|F\|_{\epsilon}$ stand for the least norm $\|M\|$ of a real matrix $M$ with $\left|F_{i j}-M_{i j}\right| \leqslant \epsilon$ whenever $F_{i j}= \pm 1$, and $\left|M_{i j}\right| \leqslant 1+\epsilon$ whenever $F_{i j}=*$. This is an instantiation for matrices of an earlier definition. The primary case of interest to us will be $\gamma_{2, \epsilon}(F)$.

Communication complexity. For an excellent exposition of quantum communication complexity, see [13], [46]. Here we will mostly limit ourselves to a review of basic facts and notation. Let $f$ be a (possibly partial) Boolean function on the Cartesian product $X \times Y$ of two finite sets $X, Y$. A quantum protocol is said to compute $f$ with error $\epsilon$ if on every input $(x, y) \in \operatorname{dom} f$, the output of the protocol disagrees with the value of $f$ with probability no greater than $\epsilon$. Analogous to classical computation, the cost of a quantum protocol is the maximum number of quantum bits exchanged between the two players on any input $(x, y)$. The least cost of an $\epsilon$-error quantum protocol (with arbitrary prior entanglement) for $f$ is denoted $Q_{\epsilon}^{*}(f)$. The precise choice of a constant $\epsilon \in(0,1 / 2)$ affects $Q_{\epsilon}^{*}(f)$ by at most a constant factor, and thus the setting $\epsilon=1 / 3$ entails no loss of generality. By the communication complexity of a (possibly partial) sign matrix $F=\left[F_{i j}\right]_{i \in I, j \in J}$ will be meant the communication complexity of the associated (possi- bly partial) Boolean function $f$ on $I \times J$ given by $f(i, j)=F_{i j}$ when $F_{i j}= \pm 1$ and undefined otherwise.

We will additionally consider the setting where the quantum protocol simultaneously solves $n$ communication problems (equivalently, sign matrices) $F_{1}, F_{2}, \ldots, F_{n}$. Given $n$ input instances $\left(x_{1}, y_{1}\right), \ldots,\left(x_{n}, y_{n}\right)$, one per communication problem, the protocol is required to output a string $z \in\{-1,+1\}^{n}$ representing a guess at the vector $\left(F_{1}\left(x_{1}, y_{1}\right), \ldots, F_{n}\left(x_{n}, y_{n}\right)\right) \in\{-1,+1\}^{n}$. As before, a $(1-\sigma)$-error protocol is one whose output differs from the correct answer with probability no greater than $1-\sigma$, on any given input. The least cost of such a protocol for $F_{1}, F_{2}, \ldots, F_{n}$ is denoted $Q_{1-\sigma}^{*}\left(F_{1}, F_{2}, \ldots, F_{n}\right)$. As usual, we allow $F_{1}, F_{2}, \ldots, F_{n}$ to be partial functions (equivalently, partial sign matrices).

In the case of $n$ communication problems $F_{1}, F_{2}, \ldots, F_{n}$, it is meaningful to consider protocols that solve all but $m$ of the $n$ instances, where the ratio $m / n$ is a small constant. In other words, given $n$ input instances $\left(x_{1}, y_{1}\right), \ldots,\left(x_{n}, y_{n}\right)$, one per communication problem, the protocol is required to output, with probability at least $\sigma$, a vector $z \in\{-1,+1\}^{n}$ such that $z_{i}=F_{i}\left(x_{i}, y_{i}\right)$ for at least $n-m$ indices $i$. We let $Q_{1-\sigma, m}^{*}\left(F_{1}, F_{2}, \ldots, F_{n}\right)$ stand for the least cost of such a quantum protocol for $F_{1}, F_{2}, \ldots, F_{n}$. When referring to this formalism, we will write that a protocol "solves with probability $\sigma$ at least $n-m$ of the problems $F_{1}, F_{2}, \ldots, F_{n}$."

THEOREM 2.4 (Linial and Shraibman). Let $\Pi$ be a quantum protocol of cost $c$, with or without prior entanglement. Then the matrix $M=[\mathbf{P}[\Pi \text { accepts }(x, y)]]_{x, y}$ satisfies $\gamma_{2}(M) \leqslant 2^{c}$.

A corollary to this result is the generalized discrepancy method for quantum communication [33, Thm. 13]:

THEOREM 2.5 (Linial and Shraibman). Let $\epsilon \in(0,1 / 2)$. For all sign matrices $F$, one has $Q_{\epsilon}^{*}(F) \geqslant \frac{1}{4} \log \left\{\gamma_{2, \frac{\epsilon}{1-\epsilon}}(F)\right\}$. For all partial sign matrices $F$, one has $Q_{\epsilon}^{*}(F) \geqslant \log \left\{\gamma_{2, \frac{\epsilon}{1-\epsilon}}(F)\right\}-3$.

The quantity $\operatorname{GDM}_{\epsilon}(F)$ from the Introduction refers to the lower bound on $Q_{\epsilon}^{*}(F)$ given by Theorem 2.5. Note that the discussion in the Introduction was in terms of a different norm $\mu$ and not $\gamma_{2}$. This substitution, original to [33], is legitimate because the two norms are within a small multiplicative factor; see [30, Sec. 2.3].

Fourier transform. Consider the vector space of functions $\{-1,+1\}^{n} \rightarrow \mathbb{R}$. For $S \subseteq\{1,2, \ldots, n\}$, define $\chi_{S}:\{-1,+1\}^{n} \rightarrow$ $\{-1,+1\}$ by $\chi_{S}(x)=\prod_{i \in S} x_{i}$. Then the functions $\chi_{S}, S \subseteq$ $\{1,2, \ldots, n\}$, form an orthogonal basis for the vector space in question. In particular, every function $f:\{-1,+1\}^{n} \rightarrow \mathbb{R}$ has a unique representation of the form $f=\sum_{S \subseteq\{1,2, \ldots, n\}} \hat{f}(S) \chi_{S}$, where $\hat{f}(S)=2^{-n} \sum_{x \in\{-1,+1\}^{n}} f(x) \chi_{S}(x)$ is the Fourier coefficient of $f$ that corresponds to $\chi_{S}$. The orthogonality of $\left\{\chi_{S}\right\}$ leads to

$$
\sum_{S \subseteq\{1,2, \ldots, n\}} \hat{f}(S)^{2}=\underset{x \in\{-1,+1\}^{n}}{\mathbf{E}}\left[f(x)^{2}\right],
$$

a fact known as Parseval's identity.

\section{PREPARATORY WORK}

There is a key similarity between quantum communication and query complexity. Specifically, every efficient communication protocol, when viewed as a matrix of acceptance probabilities, resides in the convex set corresponding to matrices of low $\gamma_{2}$ norm. Every efficient query algorithm, when viewed as a function of acceptance probabilities, resides in the convex set corresponding to low-degree polynomials. In this section, we develop a number of auxiliary re- 
sults that are not affected by the nature of the convex set and are thus common to quantum communication and query complexity. This allows us to avoid a considerable duplication of effort.

\subsection{Auxiliaries for XOR lemmas}

A starting fact in our analysis is a polynomial construction. It will subsequently play a key role in finding the witness object described in the Introduction.

LEMMA 3.1. For any $\eta_{1}, \eta_{2}, \ldots, \eta_{n} \in[0,1)$, define $\mu=$ $\Pi\left(\eta_{1}, \eta_{2}, \ldots, \eta_{n}\right)$ and $\eta=\max \left\{\eta_{1}, \eta_{2}, \ldots, \eta_{n}\right\}$. For $k=$ $0,1,2, \ldots, n-1$, let $p_{k}:[-1,1]^{n} \rightarrow \mathbb{R}$ be the unique degree- $k$ multilinear polynomial such that $p_{k}(z)=(-1)^{k} \prod_{i=1}^{k}(|z|-i)$ for $z \in\{-1,+1\}^{n}$. Then

$$
\begin{gathered}
\underset{\mu}{\mathbf{E}}\left[\left|p_{k}(z)\right|\right] \leqslant p_{k}\left(1^{n}\right) \mu\left(1^{n}\right)\left\{1+\left(\begin{array}{c}
n \\
k+1
\end{array}\right) \frac{\eta^{k+1}}{(1-\eta)^{n}}\right\}, \\
\left\|\hat{p}_{k}\right\|_{1} \leqslant k !\left(\begin{array}{c}
n+k \\
k
\end{array}\right) .
\end{gathered}
$$

Furthermore, $p_{k}(z) \geqslant 0$ for all $z \in[-1,1]^{n}$ when $k$ is even.

See the full version [41] for the proof. Using the constructed polynomial $p_{k}$, we will now construct the desired witness object $\Psi_{k}$, for later use in the XOR lemmas. For now we will only establish those properties of $\Psi_{k}$ that are common to the settings of communication and query complexity.

Lemma 3.2. Fix $\epsilon \in(0,1)$. Consider a (possibly partial) Boolean function $g_{i}$ on a finite set $X_{i}(i=1,2, \ldots, n)$. Let $\psi_{i}: X_{i} \rightarrow \mathbb{R}$ be given $(i=1,2, \ldots, n)$ with

$$
\begin{aligned}
& \left\|\psi_{i}\right\|_{1}=1 \\
& \sum_{x \in \operatorname{dom} g_{i}} g_{i}\left(x_{i}\right) \psi_{i}\left(x_{i}\right)-\sum_{x \notin \operatorname{dom} g_{i}}\left|\psi_{i}\left(x_{i}\right)\right|>(1-\epsilon)\left\|\psi_{i}\right\|_{1} .
\end{aligned}
$$

For each $i$, let $f_{i}: X_{i} \rightarrow\{-1,+1\}$ be the extension of $g_{i}$ given by $f_{i}\left(x_{i}\right)=-\widetilde{\operatorname{sgn}} \psi_{i}\left(x_{i}\right)$ outside $\operatorname{dom} g_{i}$. For $k=$ $0,1,2, \ldots, n-1$, define $\Psi_{k}: \prod X_{i} \rightarrow \mathbb{R}$ by $\Psi_{k}\left(x_{1}, \ldots, x_{n}\right)=$ $p_{k}\left(\ldots, f_{i}\left(x_{i}\right) \operatorname{sgn} \psi_{i}\left(x_{i}\right), \ldots\right) \prod_{i=1}^{n} \psi_{i}\left(x_{i}\right)$, where $p_{k}$ is the degree-k polynomial from Lemma 3.1. Then for all $\delta \geqslant 0$,

$$
\begin{aligned}
& \sum_{x \in \prod \text { dom } g_{i}} \Psi_{k}(x) \prod_{i=1}^{n} g_{i}\left(x_{i}\right)-\sum_{x \notin \prod \operatorname{dom} g_{i}}\left|\Psi_{k}(x)\right|-\delta\left\|\Psi_{k}\right\|_{1} \\
& >k !\left(1-\frac{\epsilon}{2}\right)^{n}\left\{1-\delta-(1+\delta)\left(\begin{array}{c}
n \\
k+1
\end{array}\right) \frac{\left(\frac{1}{2} \epsilon\right)^{k+1}}{\left(1-\frac{1}{2} \epsilon\right)^{n}}\right\} .
\end{aligned}
$$

Proof. It is clear that $\left\langle f_{i}, \psi_{i}\right\rangle>(1-\epsilon)\left\|\psi_{i}\right\|_{1}=1-\epsilon$ for each $i$. Let $\eta_{i}=\frac{1}{2}-\frac{1}{2}\left\langle f_{i}, \psi_{i}\right\rangle$ and $\eta=\max \left\{\eta_{1}, \eta_{2}, \ldots, \eta_{n}\right\}$. Then

$$
\eta<\frac{\epsilon}{2}
$$

Let $\lambda$ be the probability distribution on $\prod X_{i}$ given by $\lambda\left(\ldots, x_{i}, \ldots\right)=\prod\left|\psi_{i}\left(x_{i}\right)\right|$. When $\left(\ldots, x_{i}, \ldots\right) \sim \lambda$, the string $\left(\ldots, f_{i}\left(x_{i}\right) \operatorname{sgn} \psi_{i}\left(x_{i}\right), \ldots\right) \in\{-1,+1\}^{n}$ is distributed according to $\mu=\Pi\left(\eta_{1}, \eta_{2}, \ldots, \eta_{n}\right)$. As a result,

$$
\begin{aligned}
\left\|\Psi_{k}\right\|_{1} & =\sum_{X_{1} \times \cdots \times X_{n}}\left|p_{k}\left(\ldots, f_{i}\left(x_{i}\right) \operatorname{sgn} \psi_{i}\left(x_{i}\right), \ldots\right)\right| \prod_{i=1}^{n}\left|\psi_{i}\left(x_{i}\right)\right| \\
& =\underset{x \sim \lambda}{\mathbf{E}}\left[\left|p_{k}\left(\ldots, f_{i}\left(x_{i}\right) \operatorname{sgn} \psi_{i}\left(x_{i}\right), \ldots\right)\right|\right] \\
& =\underset{z \sim \mu}{\mathbf{E}}\left[\left|p_{k}(z)\right|\right] .
\end{aligned}
$$

Let $D \subseteq \prod \operatorname{dom} g_{i}$ be given by $D=\prod\left\{x_{i} \in X_{i}: f_{i}\left(x_{i}\right)=\right.$ $\left.\operatorname{sgn} \psi_{i}\left(x_{i}\right)\right\}$. Then

$$
\begin{aligned}
& \sum_{x \in \prod \text { dom } g_{i}} \Psi_{k}(x) \prod_{i=1}^{n} g_{i}\left(x_{i}\right)-\sum_{x \notin \prod \text { dom } g_{i}}\left|\Psi_{k}(x)\right|-\delta\left\|\Psi_{k}\right\|_{1} \\
& \geqslant \sum_{x \in D} \Psi_{k}(x) \prod_{i=1}^{n} f_{i}\left(x_{i}\right)-\sum_{x \notin D}\left|\Psi_{k}(x)\right|-\delta\left\|\Psi_{k}\right\|_{1} \\
& \geqslant 2 \sum_{x \in D} \Psi_{k}(x) \prod_{i=1}^{n} f_{i}\left(x_{i}\right)-(1+\delta)\left\|\Psi_{k}\right\|_{1} \\
& =2 \mu\left(1^{n}\right) p_{k}\left(1^{n}\right)-(1+\delta)\left\|\Psi_{k}\right\|_{1} \\
& =2 \mu\left(1^{n}\right) p_{k}\left(1^{n}\right)-(1+\delta) \underset{z \sim \mu}{\mathbf{E}}\left[\left|p_{k}(z)\right|\right] \\
& \geqslant \mu\left(1^{n}\right) p_{k}\left(1^{n}\right)\left\{2-(1+\delta)\left(1+\left(\begin{array}{c}
n \\
k+1
\end{array}\right) \frac{\eta^{k+1}}{(1-\eta)^{n}}\right)\right\}
\end{aligned}
$$

where the last two steps use (3.7) and (3.1), respectively. In view of (3.6) and the bound $\mu\left(1^{n}\right) p_{k}\left(1^{n}\right) \geqslant k !(1-\eta)^{n}>k !(1-\epsilon / 2)^{n}$, the proof is complete.

A common operation in this manuscript is that of bounding the correlation of a given function with the elements of a given convex set. In the case of quantum query complexity, this operation is effortless because of the way polynomial multiplication is defined. More care is needed in the setting of quantum communication complexity, where this step corresponds to bounding the norm dual to the convex set. We address the latter case below.

LEMMA 3.3. Fix finite sets $X_{1}, X_{2}, \ldots, X_{n}$. Let $\mathbb{R}^{X_{1}}, \mathbb{R}^{X_{2}}, \ldots, \mathbb{R}^{X_{n}}$, and $\mathbb{R}^{\prod X_{i}}$ be normed by $\|\cdot\|$, where

$$
\begin{aligned}
& C_{1}=\max \left\{\frac{\left\|\bigotimes_{i=1}^{n} \phi_{i}\right\|^{*}}{\prod_{i=1}^{n}\left\|\phi_{i}\right\|^{*}}: \phi_{i} \in \mathbb{R}^{X_{i}} \backslash\{0\} \text { for all } i\right\}, \\
& C_{2}=\max \left\{\frac{\|\phi\|_{\infty}}{\|\phi\|}: \phi \in \mathbb{R}^{X_{i}} \backslash\{0\} \text { for some } i\right\} .
\end{aligned}
$$

For $k=0,1,2, \ldots, n$, let $\mathscr{C}_{k}$ be the convex hull of functions $\xi: \prod X_{i} \rightarrow \mathbb{R}$ of the form

$$
\xi\left(x_{1}, \ldots, x_{n}\right)=\underset{|S|=k}{\mathbf{E}}\left[\prod_{i \in S} \xi_{S, i}\left(x_{i}\right)\right],
$$

where each $\xi_{S, i}: X_{i} \rightarrow \mathbb{R}$ obeys $\left\|\xi_{S, i}\right\|_{\infty} \leqslant 1$. Then for all $\psi_{i}: X_{i} \rightarrow \mathbb{R}$ with $\left\|\psi_{i}\right\|_{1} \leqslant 1(i=1,2, \ldots, n)$,

$$
\max _{\zeta \in \mathscr{C}_{k}}\left\|\zeta \circ \bigotimes_{i=1}^{n} \psi_{i}\right\|^{*} \leqslant C_{1} C_{2}^{k} \underset{|S|=n-k}{\mathbf{E}}\left[\prod_{i \in S}\left\|\psi_{i}\right\|^{*}\right] .
$$


Proof. By convexity, it suffices to prove the claim for the functions $\xi$ in (3.10). In view of (3.8),

$$
\left\|\xi \circ \bigotimes_{i=1}^{n} \psi_{i}\right\|^{*} \leqslant C_{1} \underset{|S|=k}{\mathbf{E}}\left[\prod_{i \in S}\left\|\xi_{S, i} \circ \psi_{i}\right\|^{*} \cdot \prod_{i \notin S}\left\|\psi_{i}\right\|^{*}\right] .
$$

By duality, (3.9) is equivalent to saying that $\|\phi\|^{*} \leqslant C_{2}\|\phi\|_{1}$ for all $\phi \in \bigcup \mathbb{R}^{X_{i}}$, whence

$$
\begin{gathered}
\left\|\xi \circ \bigotimes_{i=1}^{n} \psi_{i}\right\|^{*} \\
\leqslant C_{1} C_{2}^{k} \underset{|S|=k}{\mathbf{E}}\left[\prod_{i \in S}\left\|\xi_{S, i} \circ \psi_{i}\right\|_{1} \cdot \prod_{i \notin S}\left\|\psi_{i}\right\|^{*}\right] \\
\leqslant C_{1} C_{2}^{k} \underset{|S|=n-k}{\mathbf{E}}\left[\prod_{i \in S}\left\|\psi_{i}\right\|^{*}\right] .
\end{gathered}
$$

\subsection{Auxiliaries for direct product theorems}

We now turn our attention to the setting of direct product theorems. We start with a relaxed formalization of what it means to simultaneously solve $n$ problems.

DEFINITION 3.4 (Approximants). Fix a (possibly partial) Boolean function $g_{i}$ on a finite set $X_{i}, i=1,2, \ldots, n$. A $(\sigma, m)$ approximant for $\left(g_{1}, g_{2}, \ldots, g_{n}\right)$ is any system $\left\{\phi_{z}\right\}$ of functions $\phi_{z}: \prod X_{i} \rightarrow \mathbb{R}, z \in\{-1,+1\}^{n}$, such that:

$$
\begin{gathered}
\sum_{z \in\{-1,+1\}^{n}}\left|\phi_{z}\left(x_{1}, \ldots, x_{n}\right)\right| \leqslant 1, \quad x \in \prod X_{i}, \\
\sum_{|z| \leqslant m} \phi_{\left(z_{1} g_{1}\left(x_{1}\right), \ldots, z_{n} g_{n}\left(x_{n}\right)\right)}\left(x_{1}, \ldots, x_{n}\right) \geqslant \sigma, \quad x \in \prod \operatorname{dom} g_{i} .
\end{gathered}
$$

It is straightforward to see, as we will in sections to come, that communication protocols and query algorithms that solve with probability $\sigma$ at least $n-m$ of the problems $g_{1}, g_{2}, \ldots, g_{n}$ give rise to such representations $\left\{\phi_{z}\right\}$. The representations $\left\{\phi_{z}\right\}$ that arise in that way will obey various additional properties, but we will only appeal to the above two in the proofs of our lower bounds. As the reader may have already guessed, strong direct product theorems correspond to $m=0$, whereas threshold direct product theorems correspond to $m=\beta n$ for some small constant $\beta>0$.

We now recall a result on the polynomial approximation of symmetric functions due to de Wolf [47], improving on earlier work in [39]. We only require a rather special case of de Wolf's theorem.

THEOREM 3.5 (De Wolf). Let $\alpha>0$ be a sufficiently small absolute constant. Then for all integers $m, \ell \geqslant 0$, there is a degree- $\ell$ univariate polynomial $Q_{\ell}$ with

$$
\begin{aligned}
\left|Q_{\ell}(i)-(-1)^{i}\right| \leqslant 2^{-\frac{\alpha \ell^{2}}{n}+m+1}, & i & =0,1, \ldots, m, \\
\left|Q_{\ell}(i)\right| \leqslant 2^{-\frac{\alpha \ell^{2}}{n}+m+1}, & i & =m+1, m+2, \ldots, n, \\
\left|Q_{\ell}(i)\right| \leqslant 1, & i & =0,1, \ldots, n .
\end{aligned}
$$

In words, Theorem 3.5 gives a polynomial of reasonably low degree that approximates the parity function with extremely high accuracy at the integer points in $[0, m]$ and is exponentially close to zero at the integer points in $(m, n]$. We will need the following corollary to Theorem 3.5 .

COROLlaRY 3.6. Let $\alpha$ be the absolute constant from Theorem 3.5. Then for all integers $m, \ell \geqslant 0$, there is a degree- $\ell$ sym- metric polynomial $q_{\ell}:\{-1,+1\}^{n} \rightarrow[-1,1]$ such that:

$$
\begin{array}{rlrl}
\left|q_{\ell}(z)-\prod_{i=1}^{n} z_{i}\right| & \leqslant 2^{-\frac{\alpha \ell^{2}}{n}+m+1}, & |z| \leqslant m, \\
\left|q_{\ell}(z)\right| \leqslant 2^{-\frac{\alpha \ell^{2}}{n}+m+1}, & |z|>m, \\
\left\|\hat{q}_{\ell}\right\|_{1} \leqslant\left(\begin{array}{c}
n \\
\leqslant \ell
\end{array}\right)^{1 / 2} . &
\end{array}
$$

We now derive a key technical lemma that will allow us to obtain direct product theorems for communication and query complexity (see the full version [41] for the proof).

Lemma 3.7. Consider a (possibly partial) Boolean function $g_{i}$ on a finite set $X_{i}$, for $i=1,2, \ldots, n$. Let $\psi_{i}: X_{i} \rightarrow \mathbb{R}$ be given that obeys (3.3) and (3.4). Define $f_{i}: X_{i} \rightarrow\{-1,+1\}(i=1,2, \ldots, n)$ and $\Psi_{k}: \prod X_{i} \rightarrow \mathbb{R}(k=0,1,2, \ldots, n-1)$ as in Lemma 3.2. For a given $(\sigma, m)$-approximant $\left\{\phi_{z}\right\}$ of $\left(g_{1}, g_{2}, \ldots, g_{n}\right)$, let $\Phi_{\ell}: \prod X_{i} \rightarrow \mathbb{R}$ be defined by $\Phi_{\ell}\left(x_{1}, \ldots, x_{n}\right)=$ $\sum_{z \in\{-1,+1\}^{n}} \phi_{z}\left(x_{1}, \ldots, x_{n}\right) q_{\ell}\left(\ldots, z_{i} f_{i}\left(x_{i}\right), \ldots\right) \prod_{i=1}^{n} z_{i}$, where $q_{\ell}$ is the degree-l polynomial from Corollary 3.6. Then

$$
\begin{aligned}
\left\langle\Phi_{\ell}, \Psi_{k}\right\rangle>k !\left(1-\frac{\epsilon}{2}\right)^{n} & \left\{2-\left(2-\sigma+2^{-\frac{\alpha \ell^{2}}{n}+m+1}\right) \times\right. \\
& \left.\times\left(1+\left(\begin{array}{c}
n \\
k+1
\end{array}\right) \frac{\left(\frac{1}{2} \epsilon\right)^{k+1}}{\left(1-\frac{1}{2} \epsilon\right)^{n}}\right)\right\},
\end{aligned}
$$

where $\alpha>0$ is the absolute constant from Theorem 3.5.

\section{QUANTUM COMMUNICATION}

This section is devoted to our results on quantum communication complexity. In Section 4.1, we prove XOR lemmas and direct product theorems for any approximate norm whose dual exhibits submultiplicative behavior. In the subsections that follow, we specialize our results to $\gamma_{2}$, obtaining XOR lemmas, direct product theorems, and direct sum theorems for communication complexity.

\subsection{Solution for arbitrary norms}

In what follows, $\|\cdot\| \|$ stands for any norm on Euclidean space. The results below are meaningful as long as the dual norm behaves nicely under tensor product, viz., a reasonable bound can be placed on $\left\|\otimes \psi_{i}\right\|^{*}$ in terms of $\prod\left\|\psi_{i}\right\|^{*}$. We start with an XOR lemma.

THEOREM 4.1. Fix finite sets $X_{1}, X_{2}, \ldots, X_{n}$. Let $\mathbb{R}^{X_{1}}, \mathbb{R}^{X_{2}}, \ldots, \mathbb{R}^{X_{n}}$, and $\mathbb{R}^{\Pi X_{i}}$ be normed by $\|\cdot\|$, with $C_{1}, C_{2}$ defined by (3.8) and (3.9). Fix a (possibly partial) Boolean function $g_{i}$ on $X_{i}(i=1,2, \ldots, n)$. Then for every $\epsilon, \delta \in(0,1)$ and $k=0,1,2, \ldots, n-1$, the quantity $\left\|\otimes_{i=1}^{n} g_{i}\right\|_{\delta}$ is at least

$$
\frac{\prod_{i=1}^{n}\left\|g_{i}\right\|_{1-\epsilon}}{\mathbf{E} \mid=k\left[\prod_{i \in S}\left\|g_{i}\right\|_{1-\epsilon}\right]} \cdot \frac{1-\delta-(1+\delta)\left(\begin{array}{c}
n \\
k+1
\end{array}\right) \frac{\left(\frac{1}{2} \epsilon\right)^{k+1}}{\left(1-\frac{1}{2} \epsilon\right)^{n}}}{\frac{\epsilon^{n-k}}{\left(1-\frac{1}{2} \epsilon\right)^{n}}\left(\begin{array}{c}
n+k \\
k
\end{array}\right) C_{1} C_{2}^{k}} .
$$


Proof. By Corollary 2.2, for each $i$ there exists $\psi_{i}: X_{i} \rightarrow \mathbb{R}$ with

$$
\begin{aligned}
\left\|g_{i}\right\|_{1-\epsilon}=\frac{1}{\left\|\psi_{i}\right\|^{*}} & \left\{\sum_{x_{i} \in \operatorname{dom} g_{i}} g_{i}\left(x_{i}\right) \psi_{i}\left(x_{i}\right)\right. \\
& \left.-\sum_{x_{i} \notin \operatorname{dom} g_{i}}\left|\psi_{i}\left(x_{i}\right)\right|-(1-\epsilon)\left\|\psi_{i}\right\|_{1}\right\} .
\end{aligned}
$$

In particular, the expression in braces is positive for all $i$. By homogeneity, we may assume that $\left\|\psi_{i}\right\|_{1}=1$ for all $i$. Define $\Psi_{k}: \prod X_{i} \rightarrow \mathbb{R}$ as in Lemma 3.2. By Corollary 2.2,

$$
\begin{aligned}
\left\|\bigotimes_{i=1}^{n} g_{i}\right\| \|_{\delta} \geqslant \frac{1}{\left\|\Psi_{k}\right\|^{*}}\left\{\sum_{x \in \prod \operatorname{dom} g_{i}} \Psi_{k}(x) \prod_{i=1}^{n} g_{i}\left(x_{i}\right)\right. & \\
& \left.-\sum_{x \notin \prod \operatorname{dom} g_{i}}\left|\Psi_{k}(x)\right|-\delta\left\|\Psi_{k}\right\|_{1}\right\} .
\end{aligned}
$$

In view of (3.5), it remains to prove that

$$
\left\|\Psi_{k}\right\|^{*} \leqslant k !\left(\begin{array}{c}
n+k \\
k
\end{array}\right) \epsilon^{n-k} C_{1} C_{2}^{k} \underset{|S|=n-k}{\mathbf{E}}\left[\prod_{i \in S} \frac{1}{\left\|g_{i}\right\|_{1-\epsilon}}\right] .
$$

For this, note first that (4.1) gives

$$
\left\|\psi_{i}\right\|^{*} \leqslant \frac{\epsilon\left\|\psi_{i}\right\|_{1}}{\left\|g_{i}\right\|_{1-\epsilon}}=\frac{\epsilon}{\left\|g_{i}\right\|_{1-\epsilon}}, \quad i=1,2, \ldots, n .
$$

Let $\mathscr{C}_{0}, \mathscr{C}_{1}, \ldots, \mathscr{C}_{n}$ be as defined in Lemma 3.3. In light of (3.2) and the symmetry of the polynomial $p_{k}$ from Lemma 3.1 , one has

$$
p_{k}\left(\ldots, f_{i}\left(x_{i}\right) \operatorname{sgn} \psi_{i}\left(x_{i}\right), \ldots\right) \in k !\left(\begin{array}{c}
n+k \\
k
\end{array}\right) \mathscr{C}_{k},
$$

for all Boolean functions $f_{i}$ on $X_{i}(i=1,2, \ldots, n)$. Lemma 3.3 now implies that

$$
\left\|\Psi_{k}\right\|^{*} \leqslant k !\left(\begin{array}{c}
n+k \\
k
\end{array}\right) C_{1} C_{2}^{k} \underset{|S|=n-k}{\mathbf{E}}\left[\prod_{i \in S}\left\|\psi_{i}\right\|^{*}\right],
$$

which settles (4.2) in view of (4.3).

We now prove a direct product theorem, again in the context of an arbitrary norm. More specifically, the theorem places a lower bound on the norm of any $(\sigma, m)$-approximant for a given set of functions, as formalized in the following definition.

Definition 4.2. Fix finite sets $X_{1}, X_{2}, \ldots, X_{n}$ and a norm $\|\cdot\| \|$ on $\mathbb{R}^{\prod X_{i}}$. For a (possibly partial) Boolean function $g_{i}$ on $X_{i}(i=$ $1,2, \ldots, n)$, let

$$
\left\|g_{1}, g_{2}, \ldots, g_{n}, \sigma, m\right\|=\min _{\left\{\phi_{z}\right\}} \max _{z \in\{-1,+1\}^{n}}\left\|\phi_{z}\right\|,
$$

where the minimum is over all $(\sigma, m)$-approximants $\left\{\phi_{z}\right\}$ of $\left(g_{1}, g_{2}, \ldots, g_{n}\right)$.

THEOREM 4.3. Fix finite sets $X_{1}, X_{2}, \ldots, X_{n}$. Let $\mathbb{R}^{X_{1}}, \mathbb{R}^{X_{2}}, \ldots, \mathbb{R}^{X_{n}}$, and $\mathbb{R}^{\Pi X_{i}}$ be normed by $\|\cdot\|$, with $C_{1}, C_{2}$ defined by (3.8), (3.9). Fix a (possibly partial) Boolean function $g_{i}$ on $X_{i}(i=1,2, \ldots, n)$. Then for all $\epsilon, \sigma \in(0,1)$, and all nonnegative integers $k, \ell, m$ with $k+\ell \leqslant n$, one has the following lower bound on $\left\|g_{1}, g_{2}, \ldots, g_{n}, \sigma, m\right\|$ :

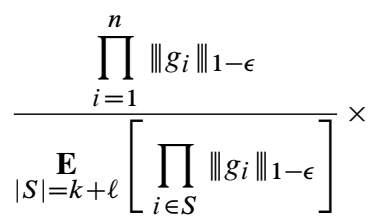

$$
\times \frac{\sigma-2^{-\frac{\alpha \ell^{2}}{n}+m+1}-2\left(\begin{array}{c}
n \\
k+1
\end{array}\right) \frac{\left(\frac{1}{2} \epsilon\right)^{k+1}}{\left(1-\frac{1}{2} \epsilon\right)^{n}}}{\frac{2^{n} \epsilon^{n-k-\ell}}{\left(1-\frac{1}{2} \epsilon\right)^{n}}\left(\begin{array}{c}
n+k \\
k
\end{array}\right)\left(\begin{array}{c}
n \\
\leqslant \ell
\end{array}\right) C_{1} C_{2}^{k+\ell}},
$$

where $\alpha>0$ is the absolute constant from Theorem 3.5.

See the full version [41] for the proof.

\subsection{XOR lemmas}

We now specialize the above results to the $\gamma_{2}$ norm and quantum communication complexity. For this, we recall a multiplicative property of the dual norm $\gamma_{2}^{*}$, established by Cleve, Slofstra, Unger, and Upadhyay [15].

TheOREM 4.4 (Cleve et al.). For all real matrices $A, B$,

$$
\gamma_{2}^{*}(A \otimes B)=\gamma_{2}^{*}(A) \gamma_{2}^{*}(B) \text {. }
$$

Theorem 4.4 was revisited more recently by Lee, Shraibman, and Špalek [31, Thm. 17], who additionally showed multiplicativity for the primal norm $\gamma_{2}$. For our purposes, only the upper bound part of Theorem 4.4 is needed. We have:

THEOREM 4.5. For all (possibly partial) sign matrices $F_{1}, F_{2}, \ldots, F_{n}$ and all sufficiently small constants $\epsilon>0$,

$$
\gamma_{2,1-\epsilon^{n / 101}}\left(\bigotimes_{i=1}^{n} F_{i}\right) \geqslant \min _{|S|=\lceil 0.99 n\rceil}\left\{\prod_{i \in S} \gamma_{2,1-\epsilon}\left(F_{i}\right)\right\} .
$$

Proof. By Theorem 4.4 and Fact 2.3 (iv), the norm $\|\cdot\|=\gamma_{2}$ satisfies (3.8) with $C_{1} \leqslant 1$ and (3.9) with $C_{2} \leqslant 1$. Hence, the result follows from Theorem 4.1 by letting $k=\lfloor 0.01 n\rfloor$ and $\delta=1-\epsilon^{n / 101}$.

Theorem 4.5 gives the desired XOR lemma for quantum communication. We will now show how to improve the dependence on the constant $\epsilon$ in the more interesting case of total functions.

THEOREM 4.6. Fix sign matrices $F_{1}, F_{2}, \ldots, F_{n}$, each of rank at least 2. Then

$\gamma_{2,1-2^{-\Omega(n)}}\left(\bigotimes_{i=1}^{n} F_{i}\right) \geqslant\left(\min _{|S|=\lceil 0.99 n\rceil}\left\{\prod_{i \in S} \gamma_{2,1 / 4}\left(F_{i}\right)\right\}\right)^{\Theta(1)}$.

See the full version [41] for the proof. This establishes the XOR results in Theorems 1.1 and 1.3 of the Introduction.

\subsection{Direct product theorems}

We will now derive direct product theorems for quantum communication, corresponding to the XOR lemmas just obtained. Recall that the symbol $Q_{1-\sigma, m}^{*}\left(F_{1}, F_{2}, \ldots, F_{n}\right)$ stands for the least cost of a quantum protocol that solves with probability $\sigma$ at least 
$n-m$ of the communication problems $F_{1}, F_{2}, \ldots, F_{n}$. The meaningful case is when the ratio $m / n$ is a sufficiently small constant. In this setting, a protocol that simply outputs a random answer $\left(z_{1}, z_{2}, \ldots, z_{n}\right) \in\{-1,+1\}^{n}$ without any communication achieves error probability $1-2^{-n}\left(\begin{array}{c}n \\ \leqslant m\end{array}\right)=1-2^{-\Omega(n)}$. All communication lower bounds below allow the protocol to err with probability $1-2^{-\Omega(n)}$.

For (possibly partial) sign matrices $F_{1}, \ldots, F_{n}$, define $\gamma_{2}\left(F_{1}, \ldots, F_{n}, \sigma, m\right)$ to be $\left\|F_{1}, \ldots, F_{n}, \sigma, m\right\|$ with $\|\cdot\|$ taken to be the $\gamma_{2}$ norm on the matrix family $\mathbb{R} \prod X_{i} \times \prod Y_{i}$.

Proposition 4.7. For all (possibly partial) sign matrices $F_{1}, \ldots, F_{n}$, one has $2^{Q_{1-\sigma, m}^{*}\left(F_{1}, \ldots, F_{n}\right)} \geqslant \gamma_{2}\left(F_{1}, \ldots, F_{n}, \sigma, m\right)$.

Proof. For a protocol $\Pi$ with cost $c$ that solves with probability $\sigma$ at least $n-m$ of the problems $F_{1}, \ldots, F_{n}$, define $\phi_{z}\left(x_{1}, \ldots, x_{n}, y_{1}, \ldots, y_{n}\right)=\mathbf{P}\left[\Pi\left(x_{1}, \ldots, x_{n}, y_{1}, \ldots, y_{n}\right)=z\right]$, where the probability is taken over the operation of the protocol on a fixed input. Then $\left\{\phi_{z}\right\}$ is a $(\sigma, m)$-approximant for $\left(F_{1}, \ldots, F_{n}\right)$. Viewed as an element of $\mathbb{R} \prod X_{i} \times \prod Y_{i}$, each $\phi_{z}$ is the matrix of acceptance probabilities of a quantum protocol with one-bit output and cost $c$ (namely, the quantum protocol that accepts if and only if $\Pi$ outputs $z$ ). Thus, $\gamma_{2}\left(\phi_{z}\right) \leqslant 2^{c}$ by Theorem 2.4 .

Recall from Theorem 4.4 and Fact 2.3 (iv) that the norm $\|\cdot\|=$ $\gamma_{2}$ satisfies (3.8) with $C_{1} \leqslant 1$ and (3.9) with $C_{2} \leqslant 1$. We will use this fact without further mention whenever we invoke our main technical tool here, Theorem 4.3. We have:

THEOREM 4.8. Fix arbitrary (possibly partial) sign matrices $F_{1}, F_{2}, \ldots, F_{n}$. Then for a sufficiently small constant $\epsilon>0$,

$$
\begin{aligned}
Q_{1-2^{-\epsilon n}, \epsilon n}^{*}( & \left.F_{1}, F_{2}, \ldots, F_{n}\right) \\
& \geqslant \epsilon \min _{|S|=\lceil 0.99 n\rceil}\left\{\sum_{i \in S} \log \left(\gamma_{2,1-\epsilon}\left(F_{i}\right)\right)\right\}-1 .
\end{aligned}
$$

Proof. A protocol that solves $\left(F_{1}, F_{2}, \ldots, F_{n}\right)$ with probability 0.99 can solve each $F_{i}$ individually with probability 0.99 . Hence, for $n$ up to any given constant, the theorem follows trivially from Theorem 2.5 by choosing $\epsilon>0$ correspondingly small. For $n$ larger than a certain constant, the theorem follows by taking $k=\ell=\lfloor 0.005 n\rfloor, m=\lfloor\epsilon n\rfloor$, and $\sigma=2^{-\epsilon n}$ in Theorem 4.3 and applying Proposition 4.7.

Theorem 4.8 gives the desired direct product theorem for quantum communication. As we did for XOR lemmas, we take a closer look at the more interesting case of total functions, improving several constants.

THEOREM 4.9. Fix sign matrices $F_{1}, F_{2}, \ldots, F_{n}$, each of rank at least 2. Then for some absolute constant $\beta>0$,

$$
\begin{aligned}
& Q_{1-2^{-\beta n}, \beta n}^{*}\left(F_{1}, F_{2}, \ldots, F_{n}\right) \\
& \geqslant \beta \min _{|S|=\lceil 0.99 n\rceil}\left\{\sum_{i \in S} \log \left(\gamma_{2, \frac{1}{4}}\left(F_{i}\right)\right)\right\} .
\end{aligned}
$$

See the full version [41] for the proof. This establishes the direct product results in Theorems 1.1 and 1.3 of the Introduction.

As a final remark, the direct product theorems in this paper can be strengthened with respect to the protocol's error probability by providing a sharper approximant than what is guaranteed in Theorem 3.5. We illustrate this point by deriving, for an arbitrarily small constant $\xi>0$, a strong direct product theorem for protocols with error probability $1-2^{-(1-\xi) n}$. This bound almost matches the error probability $1-2^{-n}$ achieved by a communication-free protocol.

THEOREM 4.10. Let $\xi>0$ be a constant and $F_{1}, F_{2}, \ldots, F_{n}$ sign matrices. Then

$$
Q_{1-2^{-(1-\xi) n}}^{*}\left(F_{1}, \ldots, F_{n}\right) \geqslant \min _{|S|=\lfloor\epsilon n\rfloor}\left\{\sum_{i \in S} \log \left(\gamma_{2,1-\epsilon}\left(F_{i}\right)\right)\right\}
$$

for a sufficiently small constant $\epsilon=\epsilon(\xi)>0$.

See the full version [41] for the proof.

\section{REMAINDER OF THIS PAPER}

See the full version [41] for the remainder of this paper, including direct sum theorems for quantum communication, all results on quantum query complexity, and all results on polynomial approximation.

\section{REFERENCES}

[1] S. Aaronson. Limitations of quantum advice and one-way communication. Theory of Computing, 1(1):1-28, 2005.

[2] S. Aaronson and Y. Shi. Quantum lower bounds for the collision and the element distinctness problems. J. ACM, 51(4):595-605, 2004.

[3] A. Ambainis. Quantum lower bounds by quantum arguments. J. Comput. Syst. Sci., 64(4):750-767, 2002.

[4] A. Ambainis, R. Špalek, and R. de Wolf. A new quantum lower bound method, with applications to direct product theorems and time-space tradeoffs. Algorithmica, 55(3):422-461, 2009.

[5] Z. Bar-Yossef, T. S. Jayram, R. Kumar, and D. Sivakumar. An information statistics approach to data stream and communication complexity. J. Comput. Syst. Sci., 68(4):702-732, 2004.

[6] B. Barak, M. Braverman, X. Chen, and A. Rao. How to compress interactive communication. In Proc. of the 42 nd Symposium on Theory of Computing (STOC), pages 67-76, 2010.

[7] R. Beals, H. Buhrman, R. Cleve, M. Mosca, and R. de Wolf. Quantum lower bounds by polynomials. J. ACM, 48(4):778-797, 2001.

[8] P. Beame, T. Pitassi, N. Segerlind, and A. Wigderson. A strong direct product theorem for corruption and the multiparty communication complexity of disjointness. Computational Complexity, 15(4):391-432, 2006.

[9] M. Bellare, O. Goldreich, and M. Sudan. Free bits, PCPs, and nonapproximability - towards tight results. SIAM J. Comput., 27(3):804-915, 1998.

[10] A. Ben-Aroya, O. Regev, and R. de Wolf. A hypercontractive inequality for matrix-valued functions with applications to quantum computing and LDCs. In Proc. of the 49th Symposium on Foundations of Computer Science (FOCS), pages 477-486, 2008.

[11] H. Buhrman, R. Cleve, R. de Wolf, and C. Zalka. Bounds for small-error and zero-error quantum algorithms. In Proc. of the 40th Symposium on Foundations of Computer Science (FOCS), pages 358-368, 1999. 
[12] H. Buhrman, I. Newman, H. Röhrig, and R. de Wolf. Robust polynomials and quantum algorithms. Theory Comput. Syst., 40(4):379-395, 2007.

[13] H. Buhrman and R. de Wolf. Communication complexity lower bounds by polynomials. In Proc. of the 16th Conf. on Computational Complexity (CCC), pages 120-130, 2001.

[14] A. Chakrabarti, Y. Shi, A. Wirth, and A. C.-C. Yao. Informational complexity and the direct sum problem for simultaneous message complexity. In Proc. of the 42nd Symposium on Foundations of Computer Science (FOCS), pages 270-278, 2001.

[15] R. Cleve, W. Slofstra, F. Unger, and S. Upadhyay. Perfect parallel repetition theorem for quantum XOR proof systems. Computational Complexity, 17(2):282-299, 2008.

[16] A. Drucker. Improved direct product theorems for randomized query complexity. Available at http://arxiv.org/abs/1005.064 4, 2010.

[17] T. Feder, E. Kushilevitz, M. Naor, and N. Nisan. Amortized communication complexity. SIAM J. Comput., 24(4):736-750, 1995.

[18] J. Håstad. Some optimal inapproximability results. J. ACM, 48(4):798-859, 2001.

[19] R. Jain and H. Klauck. New results in the simultaneous message passing model via information theoretic techniques. In Proc. of the 24th Conf. on Computational Complexity (CCC), pages 369-378, 2009.

[20] R. Jain, H. Klauck, and A. Nayak. Direct product theorems for classical communication complexity via subdistribution bounds. In Proc. of the 40th Symposium on Theory of Computing (STOC), pages 599-608, 2008.

[21] R. Jain, J. Radhakrishnan, and P. Sen. A direct sum theorem in communication complexity via message compression. In Proc. of the 30th Int'l Colloquium on Automata, Languages, and Programming (ICALP), pages 300-315, 2003.

[22] R. Jain, J. Radhakrishnan, and P. Sen. Prior entanglement, message compression and privacy in quantum communication. In Proc. of the 20th Conf. on Computational Complexity (CCC), pages 285-296, 2005.

[23] M. Karchmer, R. Raz, and A. Wigderson. Super-logarithmic depth lower bounds via the direct sum in communication complexity. Comput. Complexity, 5(3/4):191-204, 1995.

[24] H. Klauck. Quantum and classical communication-space tradeoffs from rectangle bounds. In Proc. of the 24th International Conference on Foundations of Software Technology and Theoretical Computer Science (FSTTCS), pages 384-395, 2004.

[25] H. Klauck. Lower bounds for quantum communication complexity. SIAM J. Comput., 37(1):20-46, 2007.

[26] H. Klauck. A strong direct product theorem for disjointness. In Proc. of the 42nd Symposium on Theory of Computing (STOC), pages 77-86, 2010.

[27] H. Klauck, R. Špalek, and R. de Wolf. Quantum and classical strong direct product theorems and optimal time-space tradeoffs. SIAM J. Comput., 36(5):1472-1493, 2007.

[28] I. Kremer. Quantum communication. Master's thesis, Hebrew University, Computer Science Department, 1995.

[29] E. Kushilevitz and N. Nisan. Communication complexity. Cambridge University Press, New York, 1997.
[30] T. Lee and A. Shraibman. Lower bounds in communication complexity. Foundations and Trends in Theoretical Computer Science, 3(4):263-398, 2009.

[31] T. Lee, A. Shraibman, and R. Špalek. A direct product theorem for discrepancy. In Proc. of the 23rd Conf. on Computational Complexity (CCC), pages 71-80, 2008.

[32] N. Linial, S. Mendelson, G. Schechtman, and A. Shraibman. Complexity measures of sign matrices. Combinatorica, 27(4):439-463, 2007.

[33] N. Linial and A. Shraibman. Lower bounds in communication complexity based on factorization norms. Random Struct. Algorithms, 34(3):368-394, 2009.

[34] N. Nisan, S. Rudich, and M. E. Saks. Products and help bits in decision trees. SIAM J. Comput., 28(3):1035-1050, 1999.

[35] I. Parnafes, R. Raz, and A. Wigderson. Direct product results and the GCD problem, in old and new communication models. In Proc. of the 29th Symposium on Theory of Computing (STOC), pages 363-372, 1997.

[36] R. Raz. A parallel repetition theorem. SIAM J. Comput., 27(3):763-803, 1998.

[37] A. A. Razborov. Quantum communication complexity of symmetric predicates. Izvestiya of the Russian Academy of Sciences, Mathematics, 67:145-159, 2002.

[38] R. Shaltiel. Towards proving strong direct product theorems. Computational Complexity, 12(1-2):1-22, 2003.

[39] A. A. Sherstov. Approximate inclusion-exclusion for arbitrary symmetric functions. Computational Complexity, 18(2):219-247, 2009. Preliminary version in 23rd CCC, 2008.

[40] A. A. Sherstov. The pattern matrix method. SIAM J. Comput., 2011. To appear. Preliminary version in 40th STOC, 2008.

[41] A. A. Sherstov. Strong direct product theorems for quantum communication and query complexity. In Electronic Colloquium on Computational Complexity (ECCC), March 2011. Report TR11-040.

[42] A. A. Sherstov. Communication lower bounds using dual polynomials. Bulletin of the EATCS, 95:59-93, 2008.

[43] Y. Shi and Y. Zhu. Quantum communication complexity of block-composed functions. Quantum Information \& Computation, 9(5-6):444-460, 2009.

[44] R. Špalek. The multiplicative quantum adversary. In Proc. of the 23rd Conf. on Computational Complexity (CCC), pages 237-248, 2008.

[45] E. Viola and A. Wigderson. Norms, XOR lemmas, and lower bounds for polynomials and protocols. Theory of Computing, 4(1):137-168, 2008.

[46] R. de Wolf. Quantum Computing and Communication Complexity. PhD thesis, University of Amsterdam, 2001.

[47] R. de Wolf. A note on quantum algorithms and the minimal degree of $\epsilon$-error polynomials for symmetric functions. Quantum Information and Computation, 8(10):943-950, 2008.

[48] A. C.-C. Yao. Theory and applications of trapdoor functions. In Proc. of the 23rd Symposium on Foundations of Computer Science (FOCS), pages 80-91, 1982.

[49] A. C.-C. Yao. Quantum circuit complexity. In Proc. of the 34th Symposium on Foundations of Computer Science (FOCS), pages 352-361, 1993. 